\title{
II. THE PLACE OF PLANT RESPONSES IN THE CATEGORIES OF SENSITIVE REACTIONS
}

\author{
PROFESSOR FREDERICK C. NEWCOMBE
}

UNIVERSITY oF MichigaN

More than two decades ago in the hands of Darwin, Sachs, Wiesner, Pfeffer, Strasburger, Stahl and others, the principal sensitive reactions for both fixed and freemoving plants had been determined. At that period, in animal biology only a few scattered papers had appeared on sensitive reactions. The zoologists approached this study through human psychology; starting with intelligence and reflexes in the highest animals, they cast a glance now and then toward the lower metazoa, and talked of reflexes and instinct. Plants, not standing in the line of descent which has its climax in man, were studied in an objective manner with no attempt to bring their reactions into a scheme of comparative psychology.

For the past two decades more attention has been paid to the study of behavior in the lowest animals, with the result that a great body of literature has already arisen, some of it tainted with anthropomorphism, but the most of it describing simply and carefully the reactions of the protozoa and the lower metazoa.

This independent activity on the part of the plant and animal biologists has resulted in the upbuilding of two almost independent bodies of literature on the behavior of lower organisms. Such early workers in the field as Verworn and Loeb compared and identified the reactions which they saw in the lower animals with those already published for plants; but as the zoologists have worked on, and more workers have come into the field, there has arisen among the botanists a feeling of uncertainty as to the significance of the terminology employed by the zoologists, and a hesitation in identifying plant reactions with those of the lowest animals. Thus, Francis 
Darwin, in his presidential address before the British Association for the Advancement of Science in 1908, ${ }^{1}$ intimates that he does not understand Loeb's use of the term "tropism." Botanists, probably quite generally, are in doubt as to whether Jennings's "trial and error", description can be applied to plant reactions. The existence of these two bodies of literature, and the uncertainty as to the significance of terms, calls, to my mind, for an examination of the phenomena of response in plants and in the lower animals, to the end that doubt may be removed as to the applicability of the same terms to both plant and animal response.

Proceeding to compare these two groups of organisms, we may say that both have fixed and free-moving forms. Most of the plants are fixed, and a group of their wellknown reactions which botanists call "tropisms" are exactly simulated on the animal side by hydroids, as recorded by Loeb. ${ }^{2}$ Geotropic and heliotropic bendings of the so-called stems and the so-called roots of the hydroids differ in no way from the manifestations of these phenomena in the stems and roots of plants.

Most of the lower animals are free-moving, and it is upon the phenomena of movement in these forms that the most of the animal studies have been made. Among' plants, corresponding studies have been made upon the movements of bacteria, antherozoids and the swarm-cells of algæ and fungi. A study of the literature describing the behavior of the protozoa and of the free-moving plants must convince one that the stimuli and the responses are the same in both divisions of organisms. There are animals with amœboid movements and plants with amœboid movements, both animals and plants with cilia, and both with flagella. We may go a step farther, and consider the stimuli that influence the movements of these plants and animals. We have learned that both are directed in their behavior by gravitation, light, chemicals and the electric current. The behavior in the plant

${ }^{1}$ Science, XXVIII, 353.

2 “'General Physiology," I, 103. 
group can be described by the same terms as used for the animal group.

Before proceeding farther in the comparison of plant and animal responses, we must consider the relation of responses in fixed forms to those in free-moving forms. It is well known that botanists generally use the terms "tropism" and "taxis" to distinguish movement in the fixed organisms from that in the free-moving. Some zoologists also, among them Verworn, observe the same practise. The majority of zoologists, however, following the lead of Loeb, omit the "taxis" designation wholly, and include the movements of both fixed and free-moving" forms in the term "tropism." This is merely a question of terminology, and need not occupy our time here. It is more important to consider whether the behavior of the fixed and that of the free-moving lower organisms are enough alike to be regarded as fundamentally the same. We may cite an analogy by saying that a traction engine may one hour be pulling a load along a road, and the next hour it may be stationary and operating a threshing machine. To enable it to do the one or the other requires but a slight alteration in the form of connection of its parts, a slight change in its mechanism. A swarm cell of the alga, Ulothrix, may alter its direction under the application of a slight stimulus, and swim toward the source of light. It may, a short time later, fix itself to the substratum, and now bend as a fixed organism toward the source of light. One can readily believe that the two reactions are the same except for the difference in the mechanism.

Besides the evidence for the fundamental similarity of sensitive processes in fixed and free-moving organisms furnished by the foregoing example, is the evidence presented by the quality of relation of stimulus and response in the two kinds of organisms: Both kinds of organisms, according to their structure, show simple positive or negative movements toward or away from the source of stimulation; both kinds are sensitive to the same kinds of 
stimuli, such as gravitation, light and chemicals; and both kinds vary their response according to their physiological state. In the various published observations which treat of the reactions of fixed and free-moving organisms, the authors regard the responses as belonging to the same class; and we may so regard them here. It might be said also, that the majority of reactions in the middle and higher metazoa are regarded by some as the same in kind, differing only in degree from the reactions of the protozoa. With the more complex reactions of the higher metazoa, botanists have little to do.

What now are the characteristics of these sensitive phenomena in plants and animals by which we class such phenomena together?

1. On both the plant and the protozoan side, the organisms possess no nerve cells. We used to be taught that for a reflex action there must be an afferent nerve, a ganglion and an efferent nerve. Such a chain is no longer necessary for a reflex according to definition. Pfeffer speaks of reflex acts in plants, and Massart, Jennings, Bohn and others expressly state that the tropisms of plants and nerveless animals are to be denominated reflexes, or, more specifically, non-nervous reflexes.

2. By the term "tropisms" (including" "taxims"), the botanist has understood those direction movements of plants due to external stimuli, such as gravitation, light and chemicals. But this use of the term is now too broad.

The zoologists apply the same term to similar movements of the lower animals. But not all zoologists adopt the definition of a tropism as the definition is understood by the botanists. To the botanist, "tropism" always implies a response due to what is known as the indirect action of a stimulus; that is, the stimulus produces first an excitation, the excitation sets up a wave, or impulse, which is transmitted to the reaction protoplasm, whose action gives the sensitive response. The controversy 
over the implied meaning of "tropism" as applied to animals seems to have arisen mostly through the attempts of Loeb and his followers to simplify the conception of the sensitive processes, to conceive of the stimulus as directly producing the reaction, as one might think of a heliotropic curve in a marine annelid produced directly by the sun's rays shortening the muscles on the sunward side. The controversy among the zoologists wages about the terms direct and indirect. Indeed, the banners of the opposing forces may be said to bear. respectively but these two mottoes. On the one side the herald has proclaimed these terms to the satisfaction of the botanists; that is, all sensitive reactions are indirect; that is, they are complex, the stimulus producing a change in the protoplasm, local or wide-spread, and this excitation of protoplasm producing an impulse, over a lesser or greater distance, setting in operation, or releasing, energy which brings the visible response. On the other side, the terms are but obscurely defined, and the nearest we come to a definition is that the excitation and the reaction are local.

Among plants there may be cases in which the excitation and reaction are local, and there are certainly cases in which a considerable part of the plant is affected. Rothert, experimenting with the coleoptile of the oat, found that he could induce positive heliotropism when only the basal, motile, part was illuminated, the rest being shaded; or he could obtain the same reaction when the basal part was shaded and only a millimeter of the tip of the leaf was illuminated. In the former case, for aught we know, the whole process was local; in the latter, it certainly was not local. Yet there is no doubt in our minds that the processes were the same in both cases. In both cases there were excitation, conduction, reaction; only in the latter case, the excitation was in a different part of the plant, and the conduction was over a greater stretch than in the former. Yet it is over the differences which these two illustrations show that the controversy has arisen over direct and indirect response. A case 
known to occur in plants seems to me to illustrate fairly well the so-called direct response of the zoologists: The leaves of some plants roll up when illuminated by the hot sun, due to the loss of water on the illuminated side. This is certainly direct, but most biologists would call it a mechanical, and not a sensitive response.

It has already been said that botanists accept the indirect method as applying in all cases to plants; and almost all zoologists take the same view with regard to animal reactions. We may conclude, therefore, that the term "tropism" may be used in the same sense for both plants and animals. The "tropism" resulting from direct action has not yet been demonstrated for either plants or animals. This conception of the significance of the term "tropism," as presented in the foregoing paragraphs, is not simple, but very complex; and in attempting to analyze the behavior of organisms, we meet with several conditioning phenomena, some of which may now be described. The terminology used in the following paragraphs is taken mostly from the zoologists, and botanists will note that the same terms are applicable to plant response.

One of the most widely extended of these observed phenomena is the variability of the response when the same external conditions are operating. Thus, Strasburger found the swarms-cells of Ulothrix and other alga, during their early active period, positively heliotropic, but negatively heliotropic during their later active period. The change in disposition of the peduncle of the poppr, of the Narcissus, and of the members of various other plants, is familiar to all botanists. All of the foregoing changes in disposition can be referred, for want of a clearer understanding, to internal changes incident to age. These internal conditions which influence the response to external stimulus are called also the physiological state. The foregoing examples are illustrations of the influence of physiological state on response.

The physiological state may also be altered, and hence 
the reaction of the organism may be altered, by an external agent which is not the stimulus to response. The rhizomes of Adoxa and Circcea, in normal surroundings growing horizontally because of their diageotropism, become positively geotropic when exposed to light, and grow toward the earth. The rhizomes of some other plants, in similar treatment, become negatively geotropic. Experiments have shown that these changes of direction in growth in these rhizomes are not heliotropic, but due to a change in disposition toward gravitation. Light does not cause the bending, but changes the physiological state, and gravitation causes the bending. Another illustration of a change in disposition due to external agency is furnished by the behavior of seedling peas and vetches which become more heliotropic when the atmosphere contains small quantities of illuminating gas, or of carbon monoxid. Loeb found that Volvox, some crustacean copepods and other organisms, are rendered more responsive to light by putting a weak solution of carbon dioxide, acetic or hydrochloric acid, in the water in which they are swimming. This author suggests that this influence on the physiological state ${ }^{3}$ is due to the acid preventing the formation in the organism of some anti-positive substance, normally generated. This hypothesis may not be supported by future study, but there is some evidence offered in its behalf, and it is a worthy attempt to come a step nearer to the intimate processes.

The "trial and error" hypothesis as applied by Jenning's to some phenomena of behavior in protozoa is defined by him as "the selection through varied movements of conditions not interfering with the physiological processes of the organism.", The term itself, "trial and error," has lately been abandoned by Jennings, ${ }^{5}$ but the value of the hypothesis as describing behavior he still maintains. It is plain to see that the so-called direction movements, or tropisms, as they have been generally

${ }^{3}$ Archiv f. Physiol., 115, 1906, 564.

4 "'Behavior of the Lower Organisms,', 1906.

${ }^{5}$ Amer. Naturalist, October, 1909. 
recorded for our fixed plants, can not have their behavior included in this category; for by them there is apparently no selection through varied movements. Our typical plant tropisms show that one movement or curve, and that is unvaryingly toward the assumption of the new position of equilibrium. The response is what Bohn has called resistless. The term is expressive, and may be useful; but we must not forget that all reactions of lower organisms are resistless.

There are recorded, however, the results of two series of experiments with plants which seem to me to range themselves with the phenomena described as "selection through varied movements." I refer to the establishment of half-hourly geotropic rhythm in the stems of Taraxacum and valerian, and of quarter-hourly heliotropic rhythm in the cotyledons of Phalaris and Avena, by the work of Francis Darwin and Miss Pertz. If there is doubt as to whether the rhythms just noted illustrate the first part of Jennings's hypothesis for the description of the origin of behavior, namely, "the selection through varied movements of conditions not interfering with physiological processes," there can, I believe, be no doubt that the establishment of these rhythms illustrates the second part of the hypothesis, namely, that the "resolution of one physiological state into another becomes more rapid after it has taken place a number of times." The gradual shortening of the period of oscillation, in the plants used by Darwin and Pertz, till the half-hourly or quarter-hourly rhythm was established, seems to me to express the more rapid resolution of one physiological state into another.

Though the idea of trial and error seems capable of application to but few of the recorded reactions of fixed plants, it is descriptive of the movements of bacteria, swarm-cells and spermatozoids. For these plants, by varied movements, finally arrive at a condition of the environment which does not interfere with their physiological processes. 
Another descriptive for a group of phenomena in behavior, used in both botany and zoology, but in quite different senses, is differential sensibility. Pfeffer applies this term to all of the tropistic processes of plants, including the typical tropisms and the shock-movements; and it is, as he says, true that it is only by a perception of difference in the stimulus on the two sides of an organism that we obtain any tropism. On the other hand, Nagel uses the same term-Unterschiedungsempfindung -in application to the shock-movements alone. Jennings, I believe, uses the term in Pfeffer's sense, Loeb and Bohn with nearly the same meaning as Jennings's trial and error idea, or, at least, they would claim that the trial and error phenomena are a combination of tropisms and responses to differential sensibility.

To Summarize: In considering the foregoing phenomena, only the so-called direction movements of fixed organisms and the locomotory movements of free-swimming organisms have been referred to.

The organisms considered-plants and protozoa-have no nerve-cells, but nevertheless, their reactions are, and may be, spoken of as non-nervous reflexes.

While the responses in fixed and free-moving organisms are different in their external manifestations, the relations of stimulus and response and the conditions of operation of stimulus and response are much the same in both classes of organisms.

The terminology as introduced and generally used by botanists has been chosen as mere designation of the obvious stimulus and the direction of movement of the organism. Hence tropism or taxism is used to designate all the responses which animal biologists, with closer attention to details of behavior, have subdivided into a larger number. In botany, therefore, the term tropism should be used in a more restricted sense than at present.

In both plant and animal behavior, this term may be restricted to those simple movements which result in 
placing the organism in a certain direction with reference to the direction of the stimulus.

The term fright-movement or shock-movement or recoil (or a still better one) may be used to describe those responses due to a sudden change in the environment. Some of these shock-movements are doubtless tropisms, but perhaps not all. These are the movements that are due to differential sensibility (Unterschiedungsempfindung) in the sense of Loeb, and to transition stimulation (Uebergangsreizung) in the sense of Pfeffer.

There might also be some designation for the series of responses which Jennings attempted to name as "trial and error," a complex of tropisms and shock-movements, influenced by a varying physiological state.

Very recently Bohn has proposed to account for all behavior of lower organisms under four designations: mechanical reflexes (tropisms), vital rhythms (a group of antonomous movements of the botanists), differential sensibility and associative phenomena.

The ideas contained in these terms are all illustrated by plant behavior. But the terms are inadequate, for they are not coordinate. Some of them refer to responses, some to physiological state.

Before closing, reference might be made to Semon's book, "Die Mneme," the ideas in which, especially regarding associative phenomena in behavior, aid one in gaining a possibly clearer conception of the origin of some reactions of organisms.

Besides the reactions treated in the foregoing pages, for which a harmony has been attempted between the students of plant and animal behavior, we all know that there are groups which need a better and more expressive terminology than now is in use. As an illustration of phenomena needing more precise terminology, I might refer to the reactions known as epinasty and hyponasty. A very complete and apparently adequate terminology for all sensitive, non-nervous, reactions is given by Massart in Annales de l'Institut Pasteur, 1901, and a translation of the same in Biologischem Centralblatt, 22, 1902, 9. 